\title{
Editorial
}

\section{Mudança dos critérios Qualis!}

A Associação Médica Brasileira (AMB) preocupada com o futuro das publicações científicas brasileiras, depois da divulgação dos novos critérios QUALIS da CAPES, vem desde agosto de 2009 organizando uma série de encontros em sua sede em São Paulo, em parceria com a Associação Brasileira de Editores Científicos - ABEC Brasil. Os encontros resultaram em um editorial denominado Classificação dos periódicos no sistema QUALIS da CAPES - a mudança dos critérios é URGENTE! Este foi assinado por 62 editores de revistas científicas e publicado na íntegra em todas elas e em outras inúmeras principalmente na área da saúde, evidenciando uma sensibilização e um envolvimento cada vez maior dos periódicos nacionais em discutir problemas comuns. ${ }^{1}$

A comunidade científica continua preocupada com as perspectivas, os rumos e o futuro dos periódicos brasileiros ${ }^{2,3}$. Assim, na reunião de 18 de março os editores presentes puderam avaliar as repercussões do primeiro Editorial que serviu de base para discussões em eventos e reuniões científicas pelo país. Esta última reunião contou com a participação da Dra. Lilian Caló, Coordenadora de Comunicação Científica e Avaliação do SciELO, que apresentou estudo comparativo dos periódicos brasileiros na referida base classificados por dois critérios: o primeiro conforme o fator de impacto ISI/ JCR, que usa somente as revistas indexadas na base Thomson Reuters, e segundo um índice composto pela somatória simples dos fatores de impacto ISI/ JCR e do SciELO. O fator de impacto SciELO, que também considera citações de todos os periódicos da sua base, modifica significativamente o número de citações obtidas e, consequentemente, eleva o fator de impacto dos periódicos brasileiros. Este fato ficou mais evidente com a demonstração apresentada pela Dra. Caló do ganho percentual obtido pelos periódicos com a adoção do índice composto. Fica claro que associar outros índices, criar equivalências ou alternativas diversas podem favorecer a qualificação das revistas nacionais, melhorando sua visi- bilidade e favorecendo a indexação internacional. Também deve se considerar que os pesquisadores nacionais estão preferindo publicar seus conteúdos em revistas estrangeiras ao invés de fazê-los em revistas nacionais. Esta escolha, melhora a qualificação do Programa de Pós-graduação aos quais estão inseridos, conquistam fator de impacto mais elevado e aumentam o índice $\mathrm{H}$; tudo isso única e exclusivamente por conta dos novos critérios adotados pela CAPES. A busca de maior visibilidade e qualidade da produção nacional não deve ser avaliada somente pelos artigos, mas também por maior qualificação de nossos periódicos para que eles sejam reconhecidos internacionalmente.

Considerando que para atual avaliação trienal da CAPES os critérios já estão definidos, os editores reunidos decidiram elaborar um novo editorial contendo uma lista de sugestões a ser encaminhada para a coordenação da CAPES para a próxima avaliação. A lista de sugestões que complementam a do primeiro editorial é a seguinte:

- revisão dos critérios usados pela CAPES para classificação dos periódicos, sugerindo que seja adotado o fator de impacto composto pela somatória dos fatores de impacto ISI/JCR e SciELO;

- obtenção de um assento para a ABEC Brasil (Associação Brasileira dos Editores Científicos) no Conselho Técnico Científico da CAPES, para que os editores possam ser ouvidos no processo;

- solicitação da "Bolsa do Editor" junto ao CNPq para auxílio à editoração científica destinada a editores de revistas brasileiras que recebem apoio da referida agência de fomento. Este recurso tem por objetivo aprimorar a qualidade das revistas obtendo maior dedicação de seus editores às funções editoriais.

Além disto, os editores reunidos decidiram obter apoio da Academia Brasileira de Ciências, da FINEP e do Deputado Eleuses Vieira de Paiva para suas reivindicações e sugestões. Num segundo momento

Indexada no LILACS e LILACS-Express - Latindex - DOAJ - FUNPEC-RP - SciELO 
os editores solicitarão ao $\mathrm{CNPq}$ detalhamento dos resultados e dos critérios adotados para distribuição dos recursos dos Editais para Auxílio à Editoração (AED). Com estas informações os editores pretendem construir um banco de dados com informações sobre orçamentos anuais dos periódicos brasileiros que será útil para análise comparativa e cooperação mútua. A divulgação dos dois editoriais e sua discussão continua sendo nossa meta em buscar o reconhecimento que os periódicos nacionais necessitam e merecem.

\section{REFERÊNCIAS}

1. Classificação dos Periódicos no sistema QUALIS da CAPES - A mudança dos critérios é urgente! Rev Assoc Med Bras. 2010; 56(2):127-43.

2. de Lucena AF, Tibúrcio RV. Qualis periódicos: visão do acadêmico na graduação médica. Rev Assoc Med Bras. 2009; 55(3):247-248.

3. Rocha-e-Silva Mauricio. O novo Qualis, ou a tragédia anunciada. Clinics. 2009 Jan; 64(1):1-4.

Assinam este editorial:

Adagmar Andriolo

Jornal Brasileiro de Patologia e Medicina Laboratorial

Alfredo José Afonso Barbosa

Jornal Brasileiro de Patologia e Medicina

Laboratorial

Arnaldo José Hernandez

Revista Brasileira de Medicina do Esporte

Aroldo F. Camargos

Revista Femina

Benedito Barraviera

Journal of Venomous Animals and Toxins

including Tropical Diseases

Bogdana Victoria Kadunc

Surgical \& Cosmetic Dermatology da Soc. Brasileira de Dermatologia

Bruno Caramelli

Revista da Associação Médica Brasileira

Carlos Brites

Brazilian Journal of Infectious Diseases
Dejair Caitano do Nascimento

Hansenologia Internationalis

Domingo M. Braile

Revista Brasileira de Cirurgia Cardiovascular

Dov Charles Goldenberg

Revista Brasileira de Cirurgia Plástica

Edmund Chada Baracat

Revista da Associação Médica Brasileira

Edson Marchiori

Revista Radiologia Brasileira

Eduardo de Paula Vieira

Revista Brasileira de Coloproctologia

Eros Antônio de Almeida

Revista da Sociedade Brasileira de Clínica Médica

Flávia Machado

Revista Brasileira de Terapia Intensiva

Geraldo Pereira Jotz

Arquivos Internacionais de Otorrinolaringologia

Revista Brasileira de Cirurgia Cabeça e Pescoço

Gianna Mastroianni Kirsztajn

Jornal Brasileiro de Nefrologia

Gilberto Camanho

Revista Brasileira de Ortopedia

Gustavo Gusso

Medicina Família e Comunidade

Ivomar Gomes Duarte

Revista de Administração em Saúde

Izelda Maria Carvalho Costa

Anais Brasileiros de Dermatologia

João Ferreira de Mello Júnior

Brazilian Journal of Otorhinolaryngology

Joel Faintuch

Revista Brasileira de Nutrição Clínica

José Antônio Baddini Martinez

Jornal Brasileiro de Pneumologia

José Eduardo Ferreira Manso

Revista do Colégio Brasileiro de Cirurgiões

José Eulálio Cabral Filho

Revista Brasileira de Saúde Materno Infantil

José Heverardo da Costa Montal

Revista da Associação Brasileira de Medicina

de Tráfego 
José Luiz Gomes do Amaral

Revista da Associação Médica Brasileira

José Luiz Martins

Archives of Pediatric Surgery

Jurandyr Moreira de Andrade

Revista Brasileira de Ginecologia e Obstetrícia

Leonardo Savassi

Revista Brasileira de Medicina de Família e

Comunidade

Luiz Augusto Casulari

Brasilia Médica

Luiz Eugenio Garcez Leme

Geriatria \& Gerontologia

Luiz Felipe P. Moreira

Arquivos Brasileiros de Cardiologia

Luiz Henrique Gebrim

Revista Brasileira de Mastologia

Marcelo Madeira

Revista Brasileira de Mastologia

Marcelo Riberto

Revista Acta Fisiátrica

Marcus Bastos

Jornal Brasileiro de Nefrologia

Mário Cícero Falcão

Revista Brasileira de Nutrição Clínica

Mario J. da Conceição

Revista da Sociedade Brasileira de

Anestesiologia

Mauricio Rocha e Silva

Revista Clinics

Milton Artur Ruiz

Revista Brasileira de Hematologia e

Hemoterapia

Milton K. Shibata

Arquivos Brasileiros de Neurocirurgia

Mittermayer Barreto Santiago

Revista Brasileira de Reumatologia

Nelson Adami Andreollo

Arquivos Brasileiros de Cirurgia Digestiva
Nivaldo Alonso

Brazilian Journal of Craniomaxilofacial Surgery

Osvaldo Malafaia

Arquivos Brasileiros de Cirurgia Digestiva

Olavo Pires de Camargo

Acta Ortopedica Brasileira

Paulo Manuel Pêgo Fernandes

São Paulo Medical Journal

Regina Helena Garcia Martins

Brazilian Journal of Otorhinolaryngology

Renato Soibelmann Procianoy

Jornal de Pediatria

Ricardo César Pinto Antunes

Revista da Sociedade Brasileira de

Cancerologia

Ricardo Fuller

Revista Brasileira de Reumatologia

Ricardo Guilherme Viebig

Arquivos de Gastroenterologia

Ricardo Nitrini

Dementia \& Neuropsychologia

Rogério Dedivitis

Revista Brasileira de Cirurgia Cabeça e Pescoço

Ronaldo Damião

Urologia Contemporânea

Rosângela Monteiro

Revista Brasileira de Cirurgia Cardiovascular

Sergio Lianza

Revista Medicina de Reabilitação

Sigmar de Mello Rode

Brazilian Oral Research

Tarcisio E.P. Barros Filho

Acta Ortopedica Brasileira

Wallace Chamon

Arquivos Brasileiros de Oftalmologia

Winston Bonetti Yoshida

Jornal Vascular Brasileiro

Zuher Handar

Revista Brasileira de Medicina do Trabalho 